ratio had returned to normal by the 14th week of treatment.

The value of the radioactive bromide partition test in excluding tuberculous meningitis is illustrated by the following three cases.

Case 13.-This 19-year-old English man was admitted to hospital with a five-day history of sore throat, headache, vomiting, and meningism. He was ill-looking, anaemic, and had signs of meningitis. C.S.F. on admission showed 36 white cells $/ \mathrm{mm}^{3}(70 \%$ polymorphs $30 \%$ lymphocytes) and $53 \mathrm{mg} / 100 \mathrm{ml}$ sugar and $80 \mathrm{mg} / 100$ $\mathrm{ml}$ protein. He remained ill and febrile, and a repeat C.S.F. a week later showed some deterioration, with 145 white cells $/ \mathrm{mm}^{3}(90 \%$ lymphocytes) and $42 \mathrm{mg} / 100 \mathrm{ml}$ sugar and $158 \mathrm{mg} / 100 \mathrm{ml}$ protein. The possibility of tuberculous meningitis was then considered, but as the bromide ratio at that time was $2 \cdot 2$ chemotherapy was withheld, and thereafter he improved rapidly. An echovirus type 4 was later isolated from the C.S.F. and guinea-pig inoculation was negative.

Case 14.-A 16-year old English boy was admitted with a twoweek history of backache, headache, vomiting, and abdominal pains. $\mathrm{He}$ had signs of meningitis. C.S.F. on admission contained 245 white cells $/ \mathrm{mm}^{3}$ (84\% lymphocytes) and $49 \mathrm{mg} / 100 \mathrm{ml}$ sugar and $146 \mathrm{mg} / 100 \mathrm{ml}$ protein. Although initially regarded as a case of virus meningitis, the possibility of tuberculosis infection arose when he remained ill and febrile after five days in hospital. Repeat C.S.F. showed a further rise in protein $(200 \mathrm{mg})$ and a depression of the sugar level $(33 \mathrm{mg} / 100 \mathrm{ml}$ ). The bromide ratio, however, was 2.5 and antituberculous treatment was withheld. He then recovered rapidly and uneventfully.

Case 15.-A 46-year-old Pakistani man was admitted with a sixday history of headache, vomiting, and photophobia. He also complained of persistent cough for seven months. Admission C.S.F. showed 285 white cells $/ \mathrm{mm}^{3}$ (90\% lymphocytes), $42 \mathrm{mg} / 100 \mathrm{ml}$ sugar and $142 / 100 \mathrm{ml}$ protein. The possibility of tuberculous infection was considered but a radioactive bromide partition test gave a ratio of 1.7. No treatment was given and he made a rapid and complete recovery.

Each of the above three patients gave rise to diagnostic difficulty because of such features as persistent illness and fever, usually high C.S.F. protein levels, or depressed C.S.F. sugar levels, and all illustrate the value of the radioactive bromide partition test in helping to exclude the possibility of tuberculous meningitis.

\section{Conclusions}

Our findings illustrate the value of the radioactive bromide partition test in the differential diagnosis of lymphocytic meningitis. Using the figure of 1.6 as the critical bromide ratio value we found the test $100 \%$ accurate in both the tuberculous and non-tuberculous groups, though admittedly the numbers of cases were not large. The test is simpler and seems to be more accurate than the time-consuming chemical method of bromide estimation, with which Taylor et al. ${ }^{7}$ and Nicol and Fawns ${ }^{9}$ obtained $7 \%$ and $10 \%$ falsepositives respectively in patients with non-tuberculous lymphocytic meningitis. Since treatment with antituberculous drugs does not affect the bromide ratio in the early stages chemotherapy can begin immediately in highly suspicious cases without affecting the diagnostic value of the test. Hence we think that the radioactive bromide partition test is a valuable additional procedure for the early diagnosis of tuberculous meningitis and merits further study and wider application.

We are grateful to Dr. J. Shafer, consultant physician, Burnley General Hospital, for referring Cases 3 and 10. Our thanks are also due to Dr. Parbati Sengupta and Sister Kathleen Ruddy, of Monsall Hospital, Manchester, for their invaluable help.

Reprint requests should be sent to Dr. B. K. Mandal, Monsall Hospital, Manchester.

\section{References}

${ }^{1}$ Bazin, C., Blayo, M. C., Gandebuut, C., and Emile, E., Press Médicale, $1969,77,625$.

Wiseman, R. A., and Mahmood, A., British fournal of Clinical Practice, 1967, 21, 13.

3 Kocen, R. S., and Parsons, M., Quarterly fournal of Medicine, 1970, 39, 17.

4 Wilfert, C. M., New England fournal of Medicine, 1969, 280, 855

5 Stevenson, J., and Mandal, B. K., unpublished data, 1969.

6 Walter, F. K., Die Blut-Liquorschranke. Leipzig, Thieme, 1929.

7 Taylor, L. M., Smith, H. V., and Hunter, G., Lancet, 1954, 1, 700

${ }^{\circ}$ Smith, H. V., Taylor, L. M., and Hunter, G., fournal of Nenrology, Neurosurgery, and Psychiatry 1955, 18, 237 .

9 Nicol, V. S., and Fawns, H. T., Archives of Disease in Childhood, 1958, 33, 440 .

10 Crook, A., Duncan, H., Gutteridge, B., and Pallis, C., British Medical Fournal, 1960, 1, 704.

${ }^{11}$ Mouchette, R., Merchie, G., and Chantraine, J. M., Revué Médicale de Liège, 1966, 21, 181.

\title{
Arthritis as an Engineering Problem: Leeds and London
}

\author{
FROM A SPECIAL CORRESPONDENT
}

British Medical fournal, 1972, 4, 415-417

This is the first of an occasional series of articles in which a special correspondent will describe the work of units in which medical research is closely combined with other scientific disciplines.

The bones and joints are the most obviously mechanical parts of the body, so that orthopaedics and rheumatology are logical points of contact between mechanical engineering and medicine. Indeed the early research done in the Biomechanics Unit at Imperial College, London, was on the strength and stiffness of the metal fixation devices used by orthopaedic surgeons in the management of fractures. This work was then extended to a study of the mechanical properties of bone, including its fatigue characteristics but-as was perhaps inevitable in a unit whose medical partner spends so much time dealing with patients with arthritis-the current major preoccupation at the Imperial College unit is a hypothesis of the pathogenesis of osteoarthritis.

Dr. S. A. V. Swanson and Mr. M. A. R. Freeman-respectively engineer and surgeon-take the view that the primary defect in degenerative arthritis is a disturbance in the loadcarrying function of the articular cartilage. It has been known for many years that there is a reduction in the mucopolysaccharide content of cartilage in arthritic joints, and it may be, they suggest, that this biochemical change occurs early on. Whatever the cause of the mucopolysaccharide depletion, it is accompanied by a reduction in the mechanical strength and stiffness of the cartilage. Another possibility, now being examined in the Unit, is that the cartilage suffers fatigue failure 
which, by physically disrupting the structure of cartilage, leads to an increased mobility of the mucopolysaccharides. Loss in tensile strength of such cartilage has already been clearly demonstrated in studies in the Imperial College Unit. The loss of mechanical strength in the cartilage inevitably leads to its localized collapse under stress, they suggest; and once there are localized areas of collapse the cartilage can no longer carry out its vital function of distributing stress over the whole joint surface. Uneven distribution of stresses, particularly when applied cyclically as tends to happen in joints in the legs, then leads to fatigue fractures in the trabecular bone just beneath the joint surface. Certainly this last stage-trabecular fractures -has been shown to occur and the incidence of trabecular fractures correlates well with the severity of the arthritis.

Much of the research is concerned with developing this hypothesis. There are four post-doctoral research workers in the unit, which is currently largely financed by the Medical Research Council (which has just renewed its grants for another five years). Other major contributions are made by the Department of Health and the Arthritis and Rheumatism Council and, in the past, by the Science Research Council. Inevitably most of the laboratory research has to be performed on frozen specimens, but an extensive range of tests done on specimens of bone and cartilage taken fresh from the operating theatre failed to show any significant differences in their physical and mechanical properties from those of refrigerated material. Current studies include examination of the mechanical properties of cartilage on the head of the femur, which is measured by indentation tests at sites widely distributed over its surface. The results of these indentation studies are then correlated with others concerned with the histological changes in the cartilage and its electro-chemical properties. A new section of the unit's laboratories is developing the use of radioactive trace elements in studies of the permeability and diffusion characteristics of cartilage. A whole range of new physiological data is being assembled on the basis of these studiesbut it is an essential base for the studies of arthritic joints that can then follow.

At Leeds the Bioengineering Group is also concerned with joints, but in contrast to the compact research group at Im-

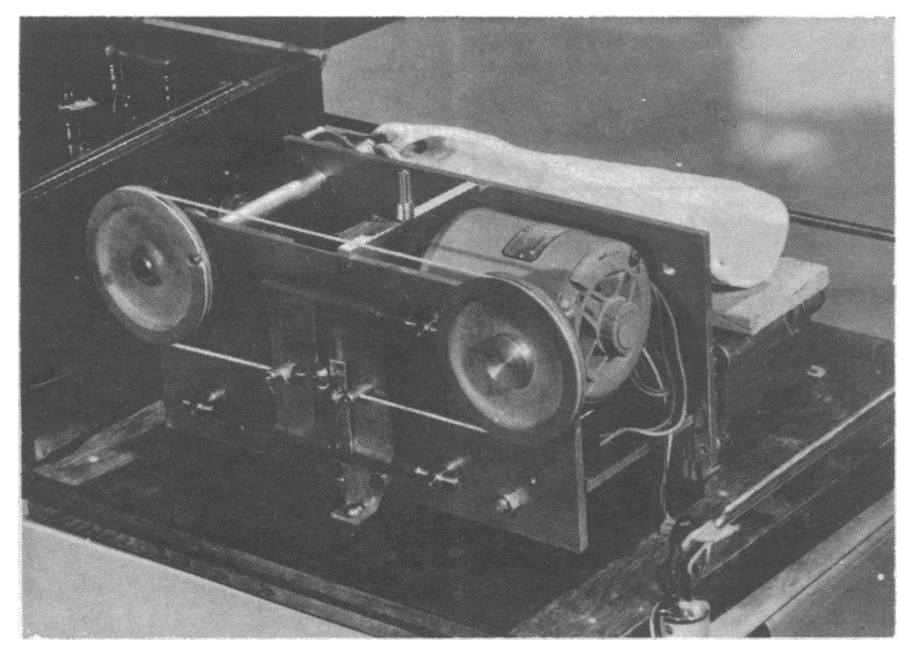

Apparatus designed at Leeds Bioengineering Unit for measuring stiffness in finger joints.

perial College the Leeds group is an informal consortium made up from members of the Rheumatism Research Unit and the University Department of Mechanical Engineering, metallurgists, biochemists, orthopaedic surgeons, a physical educationalist, a veterinary surgeon, and the doctor to Leeds United football club. Any university scientist with an interest in bioengineering problems is welcome to attend the group's meet- ings, held once a month to discuss the progress of work in hand and any new projects that might be started; the chair is taken alternately by Professor Verna Wright, a rheumatologist, and Professor D. Dowson, an engineer with a special interest in tribology, the study of friction and wear. The wide range of disciplines concerned in the group's activities is matched by the number of sources that finance them. The Science Research Council, the Arthritis and Rheumatism Council, the Medical Research Council, the West Riding Medical Trust, Reckitt and Coleman, and university research funds all provide money.

\section{Research Interests}

The group has three long-term research interests: a study of the stiffness of joints, an investigation of the pathogenesis of osteoarthritis, and the development of replacements for joints. The doctors and engineers engaged on these projects work from a common base shared with the Rheumatism Research Unit in a large Victorian house-one of many taken over by the university and scheduled for demolition within a few years as part of its massive rebuilding schemes. Within the offices there is close daily contact between the two sciences. The engineers in the group are encouraged to attend clinical ward rounds and outpatient sessions as well as preclinical physiology classes, so that they quickly become familiar with medical terminology and concepts. In addition, as each new engineering recruit joins the group he is given a small shortterm research project to give him practical experience of the problems of biological research. ("I didn't know standard deviations could be so enormous," said one engineer.) These initial projects have in fact yielded interesting results; the study undertaken by the engineer lecturer currently working in the group was on the causation of "cracking" in finger joints, which he showed to be due to sudden formation of gas bubbles-cavitation-as the pressure within the joint dropped.

Much more long-term is the research being done in the laboratories by the group of the Engineering Department on degeneration and frictional wear of cartilage and on joint lubrication. Hip joints are removed from cadavers and incorporated into a pendulum test-rig which can then be used to investigate their frictional characteristics at different loadings and with different lubricant fluids. Synovial fluid taken from cattle at the Leeds City Abattoir as well as from human patients is the subject of extensive research in the Institute of Tribology, where it is being analysed by techniques developed in the study of industrial lubricants. Eventually the institute hopes it may be able to develop an alternative lubricant to synovial fluid which might be more satisfactory in cases where the joint surfaces are badly worn.

\section{Practical Research}

Both units are also concerned with research for more obvious immediate clinical benefit, however. Early in its existence the Imperial College Unit began work on an artificial knee. The first decision that had to be made was the choice of materials -metal on metal or metal on polyethylene. It is perhaps indicative of the approach taken at Imperial College that this question was answered by a small piece of original research. Cobalt-chrome, the most satisfactory alloy for joint prostheses of which both components are metallic, might theoretically be expected to be a potentially carcinogenic material (since cobalt is known to be carcinogenic in miners). So particles of cobalt-chrome produced by laboratory wear tests on a metalon-metal joint were taken to Cambridge where Mr. J. C. Heath introduced them into the thigh muscles of rats. Given in large 
dosage the particles proved to be carcinogenic-in rats-and this finding was enough to tip the balance already in favour of a polyethylene component. Polyethylene has good frictional characteristics, and the success of the Charnley total hip replacement was sound evidence of the durability of the material. The Freeman-Swanson knee is a deceptively simple looking pad of polyethylene fixed to the top of the tibia, on which rests a light cylindrical replacement for the femoral condyles.

More than 100 of these total knee replacements have now been inserted, and more than 10 centres have been shown the technique of insertion by Mr. Freeman. The early joints have now been in patients for two years and results are so far good -even though all these subjects had advanced disease. One advantage for this knee is that so little bone has to be removed that an arthrodesis remains a possible alternative should the prosthesis fail for any reason.

At Leeds too, the group has been concerned with development of an artificial knee joint, but their main attention has been on the shoulder. Two local orthopaedic surgeons joined the consortium for this project. The total replacement of the shoulder joints was designed (with materials similar to the Charnley prosthesis for total hip replacement) with a metallic ball and plastic socket. In contrast to the normal shoulder, the ball is attached to the scapula and the socket to the humerus. Twelve of these joints have now been inserted by $\mathrm{Mr}$. Brian Reeves in patients with malignant destruction of bone or severe arthritis, and results have been good. The whole project-from initiation to production of a prosthesis for insertion -took only 18 months.

\section{Future Prospects}

More than 60 publications have come from these two units in the last few years, but the scope for future work is recognized as enormous. The recent explosion of knowledge in the biological sciences has been paralleled by similar growth in physical scientific knowledge, and one lesson that comes from units such as these is that more interchange of ideas is vital if problems are to be solved. It has, however, become clear that no matter how much research workers try to keep their work orientated to practical applied goals they find time and time again that fundamental knowledge is just not available. In addition to these basic physiological investigations there are also a whole range of clinically relevant biomechanical questions to be answered. At Imperial College, for example, the unit is interested in the contact area in various joints and how this varies from person to person. The Leeds shoulder project found that despite the apparent similarity to the hip (which has been the subject of vast amounts of biological and engineering research) very little was known of the forces acting at the shoulder because no-one had studied them. Enthusiasm for this sort of work seems widely available, and it also seems that biomechanical units can usually obtain financial support for their research projects. The only note of pessimism came from Dr. Swanson at Imperial College; his unit is finding difficulty in getting enough specimens of human joints-a difficulty blamed by him on to the unfavourable current climate of public opinion about post-mortem examinations, perhaps due partly to reaction to transplants and spare-part surgery.

\section{Today's Drugs}

With the help of expert contributors we print in this section notes on drugs in common use

\section{Diazoxide}

British Medical fournal, 1972, 4, 417-418

Diazoxide, a benzothiadiazine analogue, was originally introduced as an antihypertensive agent. Nevertheless, it was quickly shown to possess appreciable hyperglycaemic properties and since 1963 it has found an increasing use in the treatment of the hypoglycaemias. The two principal merits of diazoxide in treating these cases are its effectiveness in combating hypoglycaemias of any of a wide variety of causes, and the ready reversibility of its effects on withdrawing the treatment. In this respect it differs completely from the diabetogenic agents, alloxan or streptozotocin, which can produce irreversible destruction of normal or neoplastic insulin- producing cells.

\section{Modes of Action}

There has been controversy concerning the mode of action of diazoxide. It may be effective in at last three possible ways, in raising the blood sugar level. Firstly, there is a direct effect on the $\beta$ cells so as to reduce insulin secretion due to glucose, amino-acids such as leucine, or glucagon. This effect is seen both with normal and neoplastic islets, and usually results in a definite lowering of blood insulin levels. The action on the pancreatic $\beta$ cell can be overcome by the administration of insulin-releasing sulphonylureas, and can be prevented by the use of the $\alpha$-adrenergic blocking agent, phentolamine. This effect, through an $\alpha$-adrenergic mechanism, is consistent with the lowered levels of cyclic AMP observed in the $\beta$ cell after diazoxide administration, and would be expected to produce a reduction of rates of insulin secretion, to this extent its actions resemble those of adrenaline.

Secondly, diazoxide increases catecholamine release, and its effectiveness is known to be diminished after adrenalectomy. The resulting raised levels of circulating catecholamines may also be expected to reinforce the direct effects of diazoxide on insulin secretion and may also account for the observed rise of serum levels of free fatty acids.

Finally, diazoxide may act directly on the liver to increase its rate of glucose output. This action appears to be mediated via a rise of cyclic AMP levels in the liver, and in this organ the drug is assumed to exert its principal effect on $\beta$ adrenergic receptors. Probably the effects of diazoxide seen in normal people may result from a complex interaction of its effects at three sites, with the diminution of the rate of insulin secretion being predominant. 